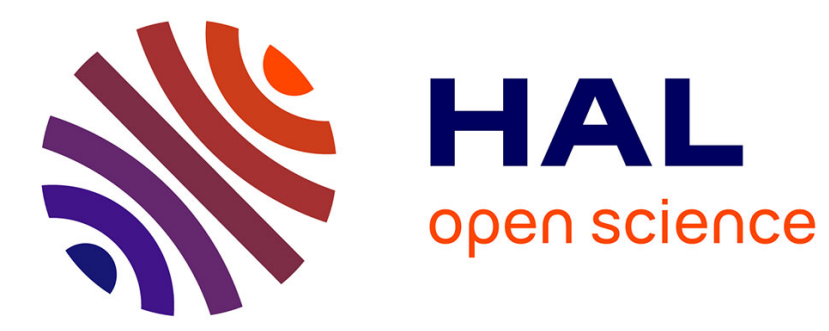

\title{
Faune et flore des niveaux profonds de Shahi-Tump (Balochistan, Pakistan) : premiers résultats
}

J Desse, Nathalie Desse-Berset, A Henry, M. Tengberg, R Besenval

\section{To cite this version:}

J Desse, Nathalie Desse-Berset, A Henry, M. Tengberg, R Besenval. Faune et flore des niveaux profonds de Shahi-Tump (Balochistan, Pakistan) : premiers résultats. Paléorient, 2009. hal-02111879

\section{HAL Id: hal-02111879 \\ https://hal.science/hal-02111879}

Submitted on 26 Apr 2019

HAL is a multi-disciplinary open access archive for the deposit and dissemination of scientific research documents, whether they are published or not. The documents may come from teaching and research institutions in France or abroad, or from public or private research centers.
L'archive ouverte pluridisciplinaire HAL, est destinée au dépôt et à la diffusion de documents scientifiques de niveau recherche, publiés ou non, émanant des établissements d'enseignement et de recherche français ou étrangers, des laboratoires publics ou privés. 


\title{
FAUNE ET FLORE DES NIVEAUX PROFONDS DE SHAHI-TUMP (BALOCHISTAN, PAKISTAN)
}

\author{
Premiers résultats
}

\author{
J. DesSe, N. DESSE-BERSET, A. HENRY, M. TENGBERG et R. BESENVAL
}

\begin{abstract}
Résumé: À Shahi-Tump, site fouillé par la Mission archéologique française au Makran (district de Kech-Makran, Balochistan pakistanais), les niveaux les plus anciens appartiennent à un faciès néolithique acéramique. Pourtant, les premiers résultats des analyses des restes archéozoologiques et archéobotaniques indiquent une séquence culturelle qui correspond à la phase ultime précédant l'introduction de la céramique dans cette région occidentale du Balochistan. L'exploitation du milieu naturel y est de faible importance et l'économie alimentaire repose fondamentalement sur des espèces animales et végétales domestiques - mais dont le spectre est encore limité. Un tel modèle est rare dans la vaste région comprise entre l'Iran oriental et l'Indus. Le seul site ayant livré des faunes et des flores domestiquées associées à des niveaux sans céramique est le site de Merhgarh. Ce dernier appartient cependant à une zone biogéographique très différente du Makran, et le rôle des animaux sauvages pour l'obtention de protéines d'origine animale y est très important, alors qu'il n'a joué qu'un rôle mineur dans l'économie de Shahi-Tump. La rareté de tels horizons dans toute cette région nous a paru justifier la diffusion des points originaux concernant l'économie alimentaire de ce site avant la publication d'autres données - tout particulièrement la métrique - qui seront présentées dans le cadre de la future monographie consacrée aux restes de ce gisement.
\end{abstract}

\begin{abstract}
At Shahi-Tump, a site excavated by the French Archaeological Mission to Makran (district of Kech-Makran, Baluchistan, Pakistan), the oldest occupational levels belong to an aceramic neolithic horizon. The first results obtained by the analysis of archaeozoological and archaeobotanical remains suggest nevertheless that we deal here with a phase immediately preceding the introduction of ceramics into this western part of Baluchistan. The exploitation of wild resources was of minor importance and the subsistence economy seems to have been mainly based on a limited number of domestic animal and plant species. This model is uncommon in the vast region stretching from eastern Iran to the Indus. The only other site that has provided remains of domestic animals and plants associated with aceramic neolithic levels is Mehrgarh. However, at this site, situated in a quite different biogeographical zone, wild animals constituted a significant part of the diet, while hunting never played an important role in the subsistence economy at Shahi-Tump. The rarity of aceramic deposits across this region justifies the publication of the first results from Shahi-Tump even though this paper does not contain detailed information, most particularly morphometric data. These will be presented at length in a future monograph dedicated to the site.
\end{abstract}

Mots-clés: Archéozoologie, Archéobotanique, Shahi-Tump, Makran, Néolithique acéramique, Paléo-environnement. Keywords: Archaeozoology, Archaeobotany, Shahi-Tump, Makran, Aceramic Neolithic, Palaeoenvironment. 


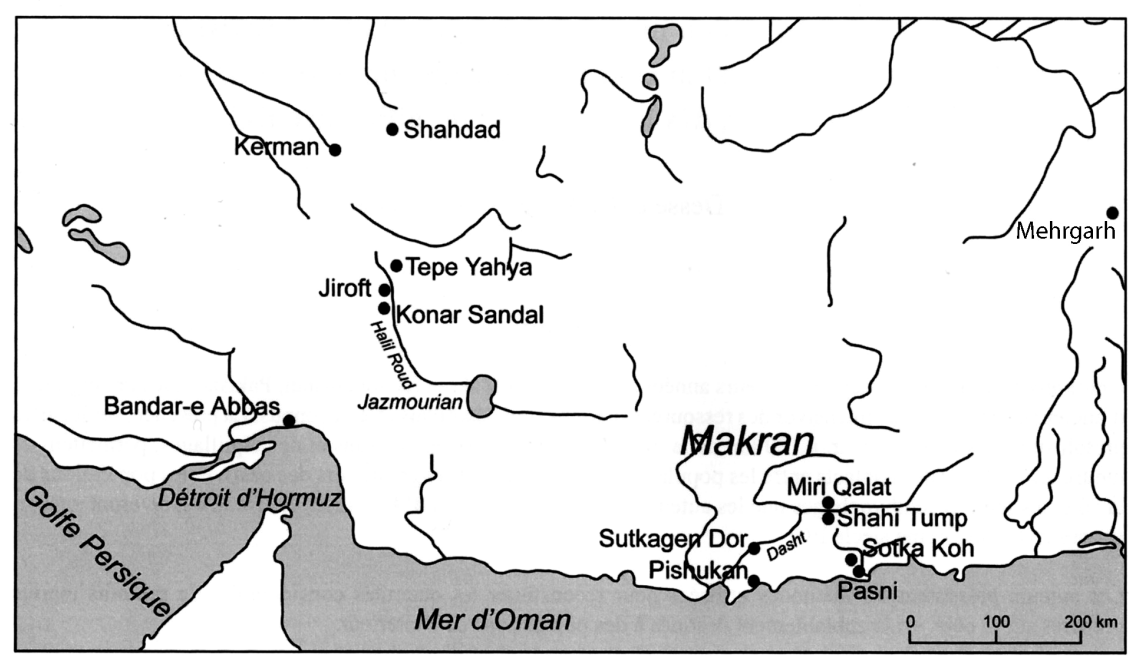

Fig. 1 - Situation géographique de Shahi-Tump.

Le peuplement protohistorique de la région comprise entre le Sud-Est iranien et le Balochistan oriental et septentrional est longtemps resté le maillon faible de notre connaissance archéologique des confins indo-iraniens. Les premières attestations de la présence d'un peuplement néolithique dans ce vaste ensemble régional remontent aux années 1960, à l'ouest dans le Kermân iranien et à l'est dans le Balochistan pakistanais, au sud de Quetta.

Dans le Kermân iranien, les travaux conduits par C. LambergKarlovsky et son équipe à partir de 1967 ont identifié à Tépé Yahya, dans la vallée de la Soghun, une occupation néolithique à la période VII de la séquence chronologique du site'. Cette période, diversement datée par les spécialistes, est généralement située entre le milieu du VI ${ }^{\mathrm{e}}$ millénaire et le milieu/fin du Ve millénaire av. J.-C.

Puis, à l'est, dans la plaine de Kachi (Balochistan pakistanais), les recherches de l'équipe dirigée par J.-F. Jarrige ont établi, à partir des sites de Mehrgarh et de Nausharo, la plus longue séquence chronologique de la Protohistoire des confins indo-iraniens ${ }^{2}$. Cette séquence commence par la mise en évidence d'une occupation néolithique acéramique, la période I, datée des $\mathrm{VIII}^{\mathrm{e}}$ et $\mathrm{VII}^{\mathrm{e}}$ millénaires. Elle est suivie d'une période IIA/B, VIe millénaire av. J.-C. qui voit, entre autres, l'apparition de la céramique.

1. LAMBERG-KARLOVSKY and BEALE, 1986; LAMBERG-KARLOVSKY and POTTS, 2001

2. JARRIGE et al., 1996 et 2005.
Cependant, entre le Kermân et la plaine de Kachi, une large zone s'étend d'ouest en est pour laquelle les informations ont longtemps manqué car, pour diverses raisons, elle n'avait fait l'objet d'aucun programme archéologique développé et continu. L'exploration d'A. Stein, en 1927-1928, avait pourtant bien souligné l'existence d'un important peuplement protohistorique dans cette partie méridionale du Balochistan ${ }^{3}$.

Dans la seconde moitié des années 1980, la Mission archéologique française au Makran (MAFM) commençait ses recherches dans la partie méridionale du Balochistan pakistanais: le Makran oriental, à partir de la ville de Turbat (district de Kech). Ces travaux ont suivi trois phases de développement:

- la première a consisté en une exploration archéologique du bassin drainant de la rivière Dasht et de la plaine côtière (de Jiwani à Ormara) qui a permis la mise en évidence de l'importance du peuplement ancien des districts de Kech et de Gwadar ${ }^{4}$.

- La deuxième phase devait combler l'absence de cadre chronologique pour la région. À ce titre, des travaux stratigraphiques furent entrepris sur le site de Miri Qalat, près de Turbat, dans la vallée de la Kech ${ }^{5}$. Outre la confirmation d'un peuplement durant la première moitié du $\mathrm{III}^{\mathrm{e}}$ millénaire (périodes IIIB et C), ainsi que la

\footnotetext{
3. STEIN, 1931 et 1937.

4. BESENVAL and SANLAVILle, 1990.

5. BESENVAL, 1997 et 2005
} 
découverte d'une occupation de la civilisation de l'Indus à Miri Qalat (période IV), ces sondages ont permis la reconnaissance d'un peuplement chalcolithique insoupçonné durant le IV millénaire av. J.-C. (périodes II et IIIa de Miri Qalat). Enfin, au fond du sondage III, une occupation ancienne, différente de celle de la période II, fut très ponctuellement atteinte (niveau $\mathrm{V}$ ); la profondeur de ce niveau rendait irréalisable toute étude ultérieure sur le site. Le niveau $\mathrm{V}$ semblait acéramique et a fourni exclusivement du matériel lithique associé à des structures en pierre. Cette occupation fut appelée période I de Miri Qalat. Un faciès semblable a été reconnu lors de prospections sur le site de Sar-i Damb situé à l'extrémité orientale du bassin drainant de la rivière Kech/Dasht.

- La troisième phase de nos travaux visait l'étude spécifique des différentes périodes chronologiques identifiées. Les résultats obtenus à Miri Qalat, pour les périodes I, II et IIIa, ont conduit à poursuivre les recherches vers le site de Shahi-Tump, situé en face de Miri Qalat, sur la rive gauche de la rivière Kech (fig. 1).

Ce site, à la suite des travaux de Stein en 1928, avait été incorrectement daté du $\mathrm{II}^{\mathrm{e}}$ millénaire av. J.-C. Cette erreur, comme nous avons pu le constater lors de nos recherches, était due à la non-observation par Stein d'un puits (ou excavation du type fosse), traversant le gisement et rempli d'un matériel tardif provenant de niveaux archéologiques rabotés par l'érosion en leur sommet. Suite aux résultats obtenus par la Mission française à Miri Qalat, nous avons pu re-dater, par le matériel céramique, l'occupation principale de Shahi-Tump d'avant le III millénaire av. J.-C.

Après plusieurs saisons de fouilles à Shahi-Tump, nous pouvons proposer le schéma chronologique suivant:

- l'occupation la plus récente (période IIIa), datée de la fin du IVe/tout début du III ${ }^{\mathrm{e}}$ millénaire, correspond à un cimetière ayant fourni un important matériel;

- la période II, datée du IV e millénaire av. J.-C., représente la phase d'occupation la plus importante du site;

- enfin, sous les niveaux de la période II, ont été atteints depuis 2005 les niveaux acéramiques de la période $\mathrm{I}$, effleurés à Miri Qalat (niveau V du chantier III) ${ }^{6}$.

Les datations radiophysiques de ce niveau, qui repose sur des poudingues, sont encore en cours, mais les informations déjà fournies par le matériel osseux et les végétaux exploités livrent des résultats originaux, inédits sur le plan régional. Un unique tesson de céramique (composé de quatre petits fragments remontant entre eux) pourrait en principe provenir de ce

6. BESENVAL, 2005. niveau ancien; en regard de ce tesson, ce même niveau nous a livré 10477 fragments osseux. Ce nombre marginalise totalement le tesson de céramique (qui est peut-être hors stratigraphie) et une telle disparité numérique est alors sans équivoque: les horizons anciens de Shahi-Tump appartiennent à un stade qui ne peut correspondre, sur le plan chrono-culturel, qu'à l'extrême fin d'un Néolithique acéramique local (ou, à l'extrême rigueur, au tout début de l'importation de céramique sur le site). Cet horizon ancien, dégagé sur plus de $120 \mathrm{~m}^{2}$ sur deux des chantiers de Shahi-Tump (chantiers I et III ${ }^{7}$ ), correspond à la période I, définie et attribuée par R. Besenval au V $V^{\mathrm{e}}$ millénaire avant notre ère ${ }^{8}$.

Cet article se propose d'explorer les données archéozoologiques et archéobotaniques afin de reconstituer l'environnement et l'économie de subsistance de cette période, à ce jour la plus ancienne occupation sédentaire identifiée dans le Makran oriental.

\section{LE CADRE ENVIRONNEMENTAL}

L'analyse de près de 4000 fragments de charbons de bois provenant des couches cendreuses à Shahi-Tump et à Miri Qalat a permis la reconstitution des formations végétales présentes dans la vallée durant la période protohistorique?.

Dès les périodes les plus anciennes, des taxons de ripisylve dominaient les assemblages anthracologiques, et les formations hygrophiles le long de la Kech constituaient la principale source de bois tout au long de l'occupation des sites. Ces formations étaient composées de tamaris (Tamarix spp.), de peupliers (Populus euphratica) et de palissandres (Dalbergia sissoo) formant de denses forêts-galeries, riches en ressources animales et végétales. Cette végétation, sans doute luxuriante, a également pu constituer une source d'alimentation pour les troupeaux domestiques, même si les pratiques de pâturage ou de collecte de fourrages n'ont pas encore été détectées dans les assemblages bioarchéologiques.

Au cours de la première moitié de l'Holocène, des conditions climatiques plus humides qu'aujourd'hui, associées à des précipitations de mousson plus soutenues, ont dû favoriser le développement des formations végétales ${ }^{10}$. Il est probable que

7. Les fouilles de ce niveau ancien ont été conduites par V. Marcon sur le chantier I et par C. Buquet sur le chantier III.

8. BESENVAL, 2005.

9. TENGBERG and ThiÉBAult, 2003.

10. Pour un résumé des études paléoclimatologiques, voir MADELla and FULLER, 2006. 
l'écoulement des cours d'eau à présent intermittents comme la Kech était alors permanent ou, du moins, s'étendait sur des périodes plus longues de l'année et permettait le développement des ripisylves. Des rivières au débit plus important et aux crues bisannuelles, liées aux précipitations d'hiver et aux pluies de mousson d'été, ont certainement eu aussi une influence bénéfique sur la pratique de l'agriculture (voir infra).

De nos jours, tandis que plusieurs espèces de tamaris caractérisent encore les bords des rivières intermittentes des vallées et plaines du Makran, le peuplier et le palissandre, espèces plus hygrophiles, ont disparu de ces écosystèmes et sont uniquement rencontrés en tant que formations résiduelles dans quelques localités particulièrement inaccessibles, notamment dans les oueds de montagne. La régression de ces espèces n'est pas un phénomène récent mais est déjà détectée dans les assemblages anthracologiques datant du $\mathrm{III}^{\mathrm{e}}$ millénaire av.J.-C. à Miri Qalat ${ }^{11}$. Elle pourrait être liée au double impact d'une aridification du climat et des activités humaines.

Parallèlement à l'exploitation de la ripisylve, du bois était collecté dans des formations ouvertes qui devaient se trouver sur des terrains non inondés, à quelque distance de la Kech. Une douzaine de taxons ligneux a été identifiée sur les deux sites. Pour les niveaux les plus anciens de Shahi-Tump, peut notamment être citée la présence de Prosopis cineraria (localement appelé kahur), un arbre de la famille des Fabacées, et le jujubier (Ziziphus sp.), qui fournissait à la fois des fruits comestibles et du bois.

La majorité des taxons présents dans la vallée de la Kech pendant la période protohistorique appartient à une flore thermophile que l'on retrouve encore, bien que fortement anthropisée, dans les confins indo-iraniens.

\section{LES FAUNES}

Le statut des faunes du niveau ancien de Shahi-Tump devient de grand intérêt en raison de l'extrême rareté des informations issues de sites de date comparable, contemporains du début de l'apparition de la céramique, pour toute la vaste zone comprise entre l'Iran oriental, l'Afghanistan méridional et l'Inde nord-occidentale. Nous ne disposons concrètement que des indications fournies par les faunes du Néolithique final de Mehrgarh ${ }^{12}$. Or, le contexte environnemental de ce gisement est très différent de celui de la région de Shahi-Tump (celui du Kech-Makran). Plus encore - et en tout premier lieu -, les résultats de l'étude archéozoologique de la période I, à Shahi-Tump, recèlent une faune domestique abondante et dominante.

Les faunes sauvages autochtones nous sont bien connues grâce aux travaux des zoologues britanniques et sont semblables à celles des marges désertiques de l'Est iranien. Le spectre faunique local est, en conséquence, bien plus restreint que celui du bassin de l'Indus; il est alors parfaitement normal que cette faible biodiversité se ressente dans tous les horizons archéologiques du Kech-Makran, surtout pour les niveaux qui sont antérieurs à la maîtrise par l'homme des techniques de sélection animale et l'introduction de nouvelles espèces. Pour les premiers niveaux antérieurs à l'introduction de la céramique dans le site, nous nous attendions à une prédation plus importante (chasse, pêche, collecte...), et à de profonds bouleversements des activités économiques. Or, les activités associées à la période I, à Shahi-Tump, ne correspondent pas au modèle attendu.

\section{LE MATÉRIEL: CONSERVATION, VALIDITÉ}

La période I, à Shahi-Tump, a livré (jusqu'aux derniers restes étudiés par nos soins: ceux de la campagne de fouille de 2006) 10477 os ou fragments pour les deux chantiers concernés (chantier I: 3338 restes; chantier III : 7139 vestiges). Le matériel atteint un haut degré de fragmentation (fig. 2) et contribue aux médiocres taux de déterminations obtenus (tabl. 1).

L'échantillon comprend plus de 1500 fragments, déterminés sur des éléments qui autorisent une identification taxonomique fine (de niveau spécifique). Ce sont ceux qui proviennent des crânes, des dents ou des os des membres et des ceintures, dont l'analyse est encore en cours, et qui fera l'objet d'une future monographie. Près de 5000 restes supplémentaires ont été déterminés au niveau générique (en prenant en compte les fragments qui proviennent d'éléments qui sont de moins bons indicateurs de niveau taxonomique). L'échantillon est ainsi numériquement assez riche pour présenter une image cohérente des faunes exploitées, et par conséquent, de l'économie des populations locales durant la période la plus ancienne du gisement.

11. TENGBERG and THIÉBAULT, 2003.

12. MEADOW, 1981. 


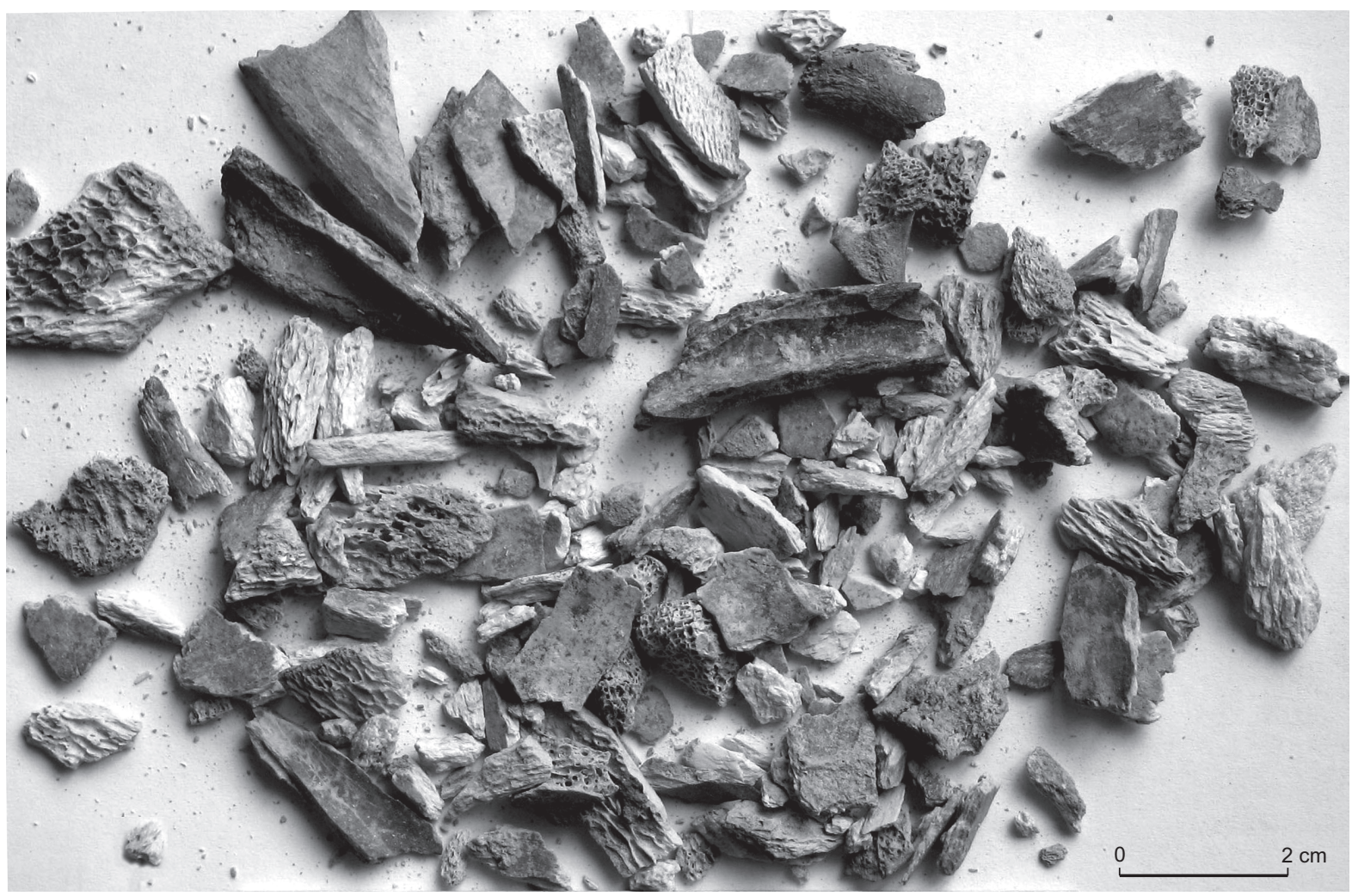

Fig. 2 - Exemple d'ossements en mauvais état de conservation (période I, chantier III, $n^{\circ} 254$ ).

Tabl. 1 - Shahi-Tump, total des faunes de la période I (chantiers I et III); représentation proportionnelle.

\begin{tabular}{|r|c|c|}
\cline { 2 - 3 } \multicolumn{1}{c|}{} & \multicolumn{1}{c|}{ NR } & $\begin{array}{c}\text { \% des } \\
\text { déterminés }\end{array}$ \\
\hline C. hircus / O. aries & 4520 & 67,70 \\
\hline Bos taurus & 1933 & 28,94 \\
\hline Total domestiques & $\mathbf{6 4 5 3}$ & \\
\hline Gazella sp. & 58 & 0,87 \\
\hline Equus hemionus & 60 & 0,90 \\
\hline Canis sp. & 3 & 0,04 \\
\hline Aves & 4 & 0,06 \\
\hline Rodentia & 2 & 0,03 \\
\hline Pisces & 5 & 0,07 \\
\hline Reptilia & 1 & 0,01 \\
\hline Mollusca & 94 & 1,41 \\
\hline Total sauvages & $\mathbf{2 2 7}$ & $\mathbf{3 , 4 0}$ \\
\hline & & en \% du total \\
\hline Indéterminés & $3 \mathbf{7 9 7}$ & 36,24 \\
\hline Total déterminés & $\mathbf{6 6 8 0}$ & $\mathbf{6 3 , 8 0}$ \\
\hline Total général & $\mathbf{1 0 ~ 4 7 7}$ & \\
\hline & &
\end{tabular}

\section{La faune sauvage}

Les os de la période I ne sont pas associés à une phase cynégétique dominante. Les animaux sauvages, petits ou gros, ne représentent qu'une faible proportion (moins de 3,5\% du nombre de restes déterminés) alors qu'une forte augmentation de la chasse, voire de la pêche, aurait pu être attendue. Les gisements étudiés par R. Meadow ${ }^{13}$ aux marges orientales du Proche-Orient fournissent également un contexte dans lequel la chasse ne joue pas un rôle important dans leur économie.

Dès la période I, les faunes mammaliennes de Shahi-Tump présentent une caractéristique fondamentale qui perdurera jusqu'à l'apparition de la civilisation de l'Indus : les activités de chasse ne jouent qu'un très faible rôle dans l'économie, avec une proportion d'ossements des mammifères sauvages (hors microfaunes) qui restera constamment inférieure à $10 \%$ des restes identifiés. Par ailleurs, le spectre faunique y est toujours restreint.

Il n'y a quasiment pas d'oiseaux dans la période I, malgré les tamisages et les flottations: 4 restes dont seuls 2 fragments attribuables à des Columbidés - leur présence relevant alors

13. MEADOW, 1986 a et 1993. 
plutôt de l'intrusif. Pourtant plusieurs mètres cube de tamisages à l'eau ont été pratiqués pour prélever les macrorestes végétaux et la microfaune. Le niveau ancien est également très pauvre en faunes marines, vertébrées comme invertébrées: il n'a livré que 5 ossements de poissons (dont 3 otolithes de petits Sciaenidae et une vertèbre d'un Chondrichthyen) et 94 fragments de coquille marines (dont 55 consistent en fragments de Dentalium et pourraient être des éléments de parure). Cette différence numérique avec tous les horizons plus récents du site est importante. Qu'il s'agisse de parure, de dépôts en contexte funéraire ou tout simplement de consommation alimentaire, les coquillages et les poissons sont abondamment représentés sur le site dès la période $\mathrm{II}^{14}$. Durant la période I de Shahi-Tump, les contacts avec la côte existent déjà, mais ils sont encore peu développés. La région de Pasni, la côte la plus proche, est à environ trois jours de marche $(120 \mathrm{~km})$ depuis Turbat.

Il n'y a que très peu de restes de carnivores: seuls trois fragments sont attribuables à de petits Canidés (chacals/renards), alors que loups, hyènes, petits Mustelidés, caracals ou autres petits félins sont encore présents de nos jours dans la région de Turbat, tout comme survivent toujours au Makran la plupart des espèces citées. Il n'y a pas d'ossements de lièvres, pas plus que de restes de tortues terrestres, de varans ou de crocodiles (ces derniers étaient présents, récemment encore, dans la Kech). Il ne figure aucun os ni aucune dent attribuable au sanglier, qui devait certainement abonder dans la vallée, son habitat naturel. Dans le cas précis des Suidés, on peut alors songer à un interdit alimentaire qui aurait affecté, au Néolithique final et durant l'âge du Bronze, toute l'Asie centrale, du Tadjikistan jusqu'au Makran ${ }^{15}$; le sanglier n'a pas été chassé, et le porc n'y a pas été domestiqué avant le passage d'Alexandre et la colonisation grecque.

De façon plus surprenante encore, nous n'avons pas mis en évidence le moindre reste de Capriné sauvage, tant d'ibex (Capra ibex L.) que de chèvre aegagre (Capra aegagrus L.) ou de mouflon (Ovis vignei L.), alors que tous ces ruminants sauvages avaient certainement leur habitat naturel privilégié dans tous les piémonts, à faible distance du site.

Alors que des bouquetins, des mouflons affrontés ou des panthères décoreront, de la plus superbe manière (et dès leur apparition), les riches céramiques de Shahi-Tump et des autres sites de la région, ce cortège faunique potentiel n'a aucune confirmation factuelle dans l'activité cynégétique concrète des groupes humains locaux, et cela dès le niveau le plus ancien du site.

14. DeSSE et DESSE-BerSET, 2005a et b; DESSE-Berset et DESSE, 2007.

15. BÖKÖNYI, 1993 ; DESSE, 1997.
Quel est alors le rôle de la prédation à l'encontre du règne animal? Il ne concerne que quelques gazelles (Gazella sp., vraisemblablement $G$. bennetti L., identifiée dans cette zone géographique à Tepe Yahya et à Mehrgahr par Meadow) et de rares hémiones (Equus hemionus L.); ces animaux sauvages ont probablement dû s'approcher imprudemment des zones cultivées, aux abords du site. Leurs os représentent, avec 118 fragments, la quasi-totalité des restes de mammifères sauvages de la période I. Les ossements attribuables à l'hémione présentent de surcroît une caractéristique fréquente et observée en de fort dissemblables régions géographiques: celle de n'être représentés que par des os de l'extrémité des membres et/ou par des fragments crâniens (dents). Nulle raison due à la conservation différentielle n'est envisageable pour interpréter ce phénomène.

$\mathrm{Ce}$ maigre tableau du spectre faunique ne peut être contourné. Les méthodes de fouille ont été particulièrement précautionneuses pour ce niveau I, et des tamisages à l'eau ont été opérés systématiquement. Ces lacunes affectent toutes les faunes mammaliennes, de la moyenne à la grande taille, depuis le lièvre jusqu'à l'aurochs asiatique. Pourtant, tous ces animaux possèdent des éléments de charpente osseuse et des dents tout à la fois robustes et bien identifiables; ils ne pouvaient échapper aux fouilleurs, ni aux spécialistes. Il nous faut alors en conclure que les anciens habitants du niveau ancien de Shahi-Tump n'ont pas éprouvé la moindre nécessité de chasser, de piéger ou de pêcher, et qu'ils étaient parfaitement satisfaits de leurs chèvres, de leur gros bétail et des multiples ressources du monde végétal qu'ils maîtrisaient déjà ${ }^{16}$. Tous les indices révélateurs du niveau alimentaire indiquent, sans nuances, une société dans laquelle les ressources en protéines animales suffisent aux besoins du groupe. Les renseignements apportés par les études archéobotaniques abondent et confortent ce modèle d'une population sans «stress» alimentaire discernable. Bien qu'encore préliminaire, cette étude des faunes de la période I de Shahi-Tump offre un échantillon numériquement suffisant et qui provient d'une superficie assez vaste $\left(120 \mathrm{~m}^{2}\right)$ pour que ces données fondamentales ne puissent être ultérieurement modifiées de manière significative par une incrémentation de l'échantillon, après une reprise des opérations de terrain et l'extension des surfaces fouillées.

\section{Les animaux domestiques (tabl. 1)}

Les ossements identifiés appartiennent aux animaux du gros et du petit bétail, à Bos taurus et Capra hircus et/ou Ovis

16. HENRY, 2003. 


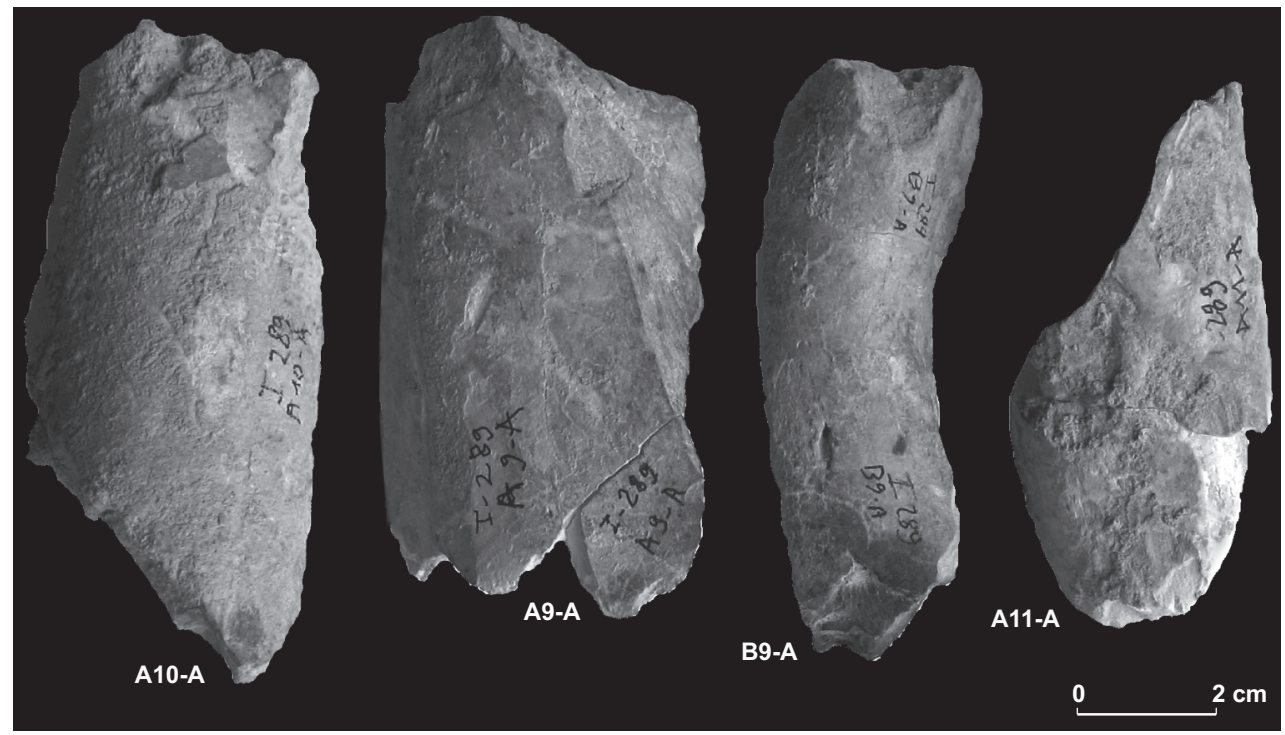

Fig. 3 - Diaphyses de Bos taurus systématiquement éclatées (période I, chantier I, $n^{\circ} 289$ ).

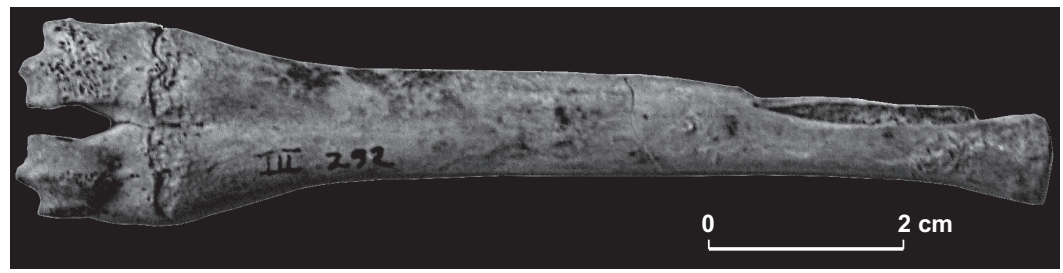

Fig. 4 - Exemple de la bonne préservation des ossements de petits ruminants; ici, un métacarpe de chèvre (période I, chantier III, $n^{\circ} 292$ ).

aries, bien que nous ne disposions pas dans les niveaux anciens de restes de moutons identifiés avec certitude. La présence de Ovis aries reste alors possible mais non certaine et, si tel est le cas, la chèvre est toujours très largement dominante parmi le petit bétail de la période I. Ainsi, aucun des fragments de chevilles osseuses du chantier I ou du chantier III ne se rapporte à Ovis. Ce déficit traduit probablement, dès les niveaux anciens du site, les grandes difficultés de l'élevage du mouton dans les conditions particulièrement arides du Makran. Tous les ossements mesurables des Caprinés de la période I offrent une caractéristique fondamentale: tous, à l'exception d'une cheville osseuse de Capra hircus L. (que sa torsion permet d'attribuer à un robuste mâle domestique), présentent des dimensions inférieures à celles des plus graciles femelles de Capra aegagrus L. ou de mouflons du Balochistan.

Le chien (Canis familiaris) est également absent des niveaux profonds de Shahi-Tump. Une semblable absence a également été notée à $\mathrm{Nal}^{17}$; le chien est cependant présent dans des niveaux plus récents de Shahi-Tump, qui sont contemporains du niveau II de Nal.

Tous les fragments du niveau ancien qui ont pu être attribués à du bœuf (Bos taurus L.) appartiennent sans le moindre doute à des individus domestiques (aucun des os mesurables n'y atteint les dimensions minimales de femelles d'aurochs). Le zébu (Bos indicus L.), également domestique, n'y est pas identifié, car aucun des os nécessaires à la diagnose, tels que crâne entier ou vertèbre dorsale, n'est attesté; sa présence ne peut toutefois pas être totalement exclue. Le buffle d'eau (Bubalus bubalis L.) est en revanche absent du site. Ces vaches et taureaux, sans être de grande taille, sont plus graciles que les bovins de l'Harappéen - mais ces derniers consistent principalement en zébus ou en demi-zébus (Bos indicus L.) -, qui ont fait l'objet, au cours des

17. BENECKE and NEEF, 2001. 
deux mille ans ou plus qui les séparent des niveaux profonds de Shahi-Tump, de considérables progrès zootechniques.

Les diagrammes obtenus par les séries de mesures déjà exploitables indiquent que les individus de la période I ne diffèrent pas, par leurs dimensions, de celles des Bos taurus des niveaux plus récents du site. Un animal du chantier III est pourtant particulièrement robuste et ses dimensions le mènent au seuil métrique des plus petites femelles d'aurochs (Bos primigenius L.). Il s'agit pourtant plus vraisemblablement d'un mâle domestique, qui correspond à un individu de taille légèrement supérieure à celle des autres mâles du gros bétail local; il faut peut-être y voir les effets d'une sélection humaine de mâles particulièrement robustes (comme la recherche d'animaux de prestige?).

\section{UNE EXPLOITATION SYSTÉMATIQUE DES OS LONGS DE BOS TAURUS?}

La dégradation la plus forte du matériel osseux affecte sélectivement les os longs de Bos taurus et elle semble surtout découler d'actions anthropiques: bris, concassage des diaphyses, etc., plutôt que d'altérations d'origine naturelle (fig. 3). Les os longs des petits mammifères sauvages ou domestiques ne présentent pas de semblables stigmates (fig. 4). De même, certains os, pourtant de forte taille, comme les astragales des bœufs, n'ont pas été brisés. Ce traitement particulier des diaphyses de gros os longs peut classiquement être associé à une recherche systématique de moelle. Ainsi, tout comme dans les sociétés qui ne disposaient pas suffisamment de protéines animales et étaient soumises à de sévères disettes - telles celles du Tardiglaciaire européen -, les phalanges proximales de Bos et tous les os contenant de la moelle sont systématiquement éclatés (fig. 5). Ce traitement particulier des os longs des grands animaux doit alors être mis en relation avec une utilisation technique particulière, plutôt qu'avec un rôle strictement alimentaire.

De toute évidence, le traitement violent opéré sur ces os n'a pas été effectué avec les silex de la période I, car ces derniers, en très faible nombre, ne consistent qu'en petits éclats subcentimétriques ${ }^{18}$. En revanche, les galets naturels de la Kech, qui sont disponibles en grande quantité autour du site, étaient parfaits pour de telles actions et leur abondance permettait de disposer d'un stock quasi-illimité de tels percuteurs.

Ce traitement systématique des os longs de Bos affecte plus de $80 \%$ des Phalanges I et II concernées et individualise l'utilisation des os de la période I à Shahi-Tump. Létude ultérieure

18. V. Marcon, communication personnelle. de l'ensemble des faunes du site, dans le cadre de la monographie générale, permettra de préciser numériquement ces premières observations.

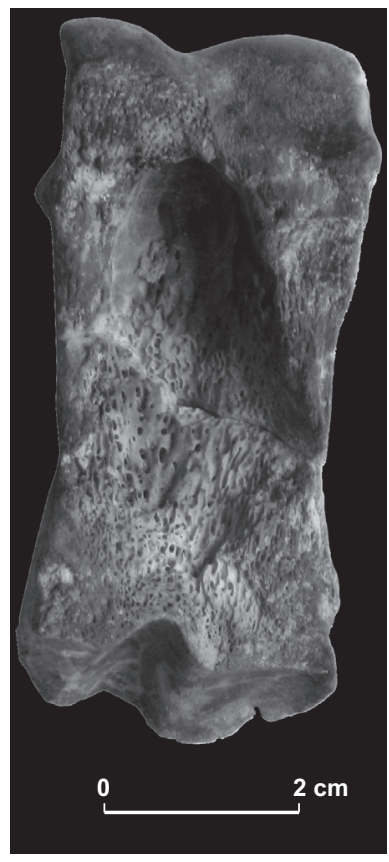

Fig. 5 - Phalange proximale de Bos taurus, éclatée pour en extraire la moelle (période I, chantier I, $n^{\circ} 303$ C23-B).

Quel était le but recherché? L'utilisation non alimentaire de la moelle est souvent associée à la préparation des cuirs et peaux. Quelques grands fragments de diaphyses, moins abîmés que les autres, évoquent un outillage osseux particulier qui requiert un biseau courbe, sorte de gouge ou de «queurçoir», peut-être destiné à travailler l'écorce de certains végétaux (fig. 6). Les enlèvements de matière ont été obtenus par de violentes percussions d'origine indubitablement anthropique. Leur bord a une délinéation régulière, quasi rectiligne, qui a été produite par des enlèvements d'amplitude variable, ce qui est caractéristique d'une mise en forme ${ }^{19}$. Cette hypothèse sera confrontée à une analyse tracéologique.

19. H. Plisson, communication personnelle. 


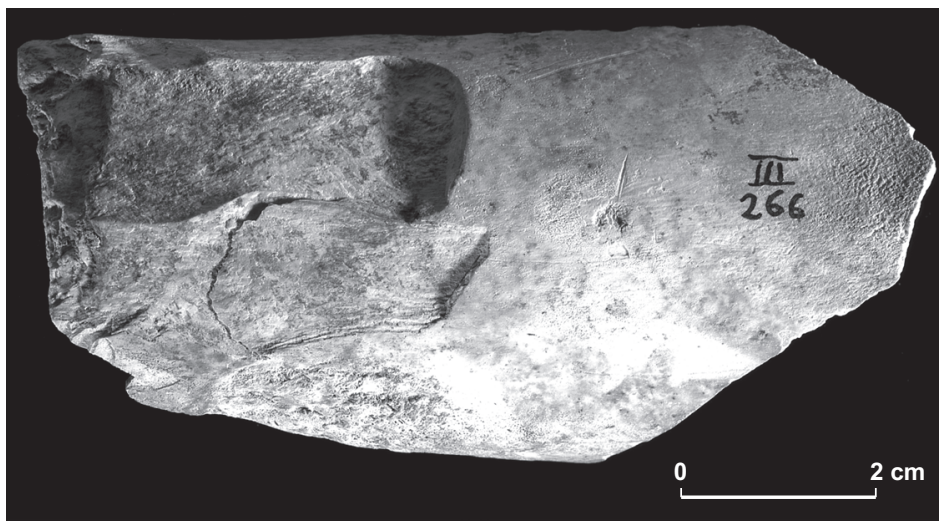

Fig. 6 - Fragment de diaphyse de bouf aménagée en outil (queurçoir?) (période I, chantier III, $n^{\circ} 266$ ).

\section{LES RÉSULTATS DE L'ANALYSE CARPOLOGIQUE}

Seuls deux sites dans le vaste espace des confins indoiraniens ont permis d'avoir un aperçu de l'économie végétale à la période néolithique: Mehrgarh dans la plaine de Kachi et Tepe Yahya dans la province iranienne du Kermân ${ }^{20}$. Dès les niveaux les plus anciens de ces gisements, qui précèdent ceux de Shahi-Tump, on note la présence de céréales cultivées : orge et blé. À Mehrgarh où l'essentiel de nos informations provient des empreintes végétales sur brique crue, plusieurs espèces de blé ont été identifiées. Tandis que la période néolithique acéramique est caractérisée par la présence de blés vêtus - engrain (Triticum monococcum) et amidonnier (T. dicoccum) -, le blé nu (T. aestivum/durum) gagne en importance au cours de l'occupation du site. Rien n'indique pour le moment qu'un processus de domestication des céréales ait eu lieu à Mehrgarh ou dans le Kermân iranien même si la présence d'orge sauvage (Hordeum spontaneum) rend théoriquement possible une origine locale de l'orge cultivée. En revanche, l'aire de distribution des blés sauvages, limitée au Proche-Orient, exclut une domestication locale. Il semble en somme que les espèces végétales cultivées dans ces régions au Néolithique soient arrivées sur les sites déjà sous leur forme domestique, à partir des foyers proche-orientaux.

20. Costantini, 1984; COSTANTINI and COSTANTINI-BIASINI, 1985; MEADOW, 1986b.

\section{MATÉRIEL ET MÉTHODES}

Seuls les niveaux inférieurs du chantier I de Shahi-Tump ont jusqu'alors fait l'objet d'une étude archéobotanique complète $^{21}$; ceux du chantier III sont encore en cours d'analyse. Tous les macrorestes botaniques (bois, fruits, graines...) de ces niveaux ont été conservés par carbonisation, résultant de leur exposition au feu, sans doute le plus souvent en relation avec des activités domestiques, notamment la préparation d'aliments.

Les restes archéobotaniques ont été extraits des sédiments par la méthode de la flottation. Nous avons utilisé une installation avec trois cuves et un système de recyclage de l'eau à l'aide d'une pompe électrique. Un tamis à maille réduite $(0,5 \mathrm{~mm})$ a été employé afin de récupérer les éléments les plus petits, notamment les graines de certaines plantes sauvages. Cette méthode a également permis de récupérer des éléments de faune de petite taille.

Les restes végétaux des niveaux les plus anciens de ShahiTump, attribués à la période I, étaient peu nombreux $(\mathrm{N}=612)$ et généralement en assez mauvais état, d'où le nombre relativement élevé de restes déterminés uniquement au niveau de la famille, voire non déterminés (tabl. 2).

\section{LES PLANTES CULTIVÉES}

À l'instar de Mehrgarh et de Tepe Yahya, les seules plantes cultivées des niveaux les plus anciens de Shahi-Tump sont des

21. HENRY, 2003 
Tabl. 2 - Résultats de l'analyse carpologique des niveaux inférieurs du chantier I, Shahi-Tump.

\begin{tabular}{|c|c|c|c|c|}
\hline \multirow[b]{4}{*}{ Catégorie } & & & & \\
\hline & \multirow[b]{3}{*}{ Nom vernaculaire } & \multirow{3}{*}{$\begin{array}{c}\text { Chantier } \\
\text { Période } \\
\text { Taxon identifié } \\
\end{array}$} & \multirow{2}{*}{\multicolumn{2}{|c|}{$\frac{\text { I (niv. inf.) }}{\text { I }}$}} \\
\hline & & & & \\
\hline & & & $\mathrm{N}$ restes & $\%$ \\
\hline \multirow{5}{*}{ Céréales } & Orge & Hordeum vulgare & 6 & 1,0 \\
\hline & Blé & Triticum sp. & 8 & 1,3 \\
\hline & Blé nu & Triticum durum/aestivum & 3 & 0,5 \\
\hline & Blé amidonnier & Triticum dicoccum & 5 & 0,8 \\
\hline & Céréales, fragments & Cerealia & 210 & 34,0 \\
\hline \multirow{4}{*}{ Graminées sauvages } & Ivraie & Lolium sp. & 1 & 0,2 \\
\hline & Alpiste & Phalaris sp. & 2 & 0,3 \\
\hline & Type Panicoïde & Panicoidae & 18 & 2,9 \\
\hline & Graminées, indéterminées & Poaceae & 8 & 1,3 \\
\hline \multirow{5}{*}{$\begin{array}{l}\text { Légumineuses } \\
\text { sauvages }\end{array}$} & Acacia/Prosopis & Acacia/Prosopis & 30 & 4,9 \\
\hline & Astragale & Astragalus sp. & 76 & 12,3 \\
\hline & Luzerne & Medicago sp. & 27 & 4,4 \\
\hline & Trèfle/Mélilot & Trifolium/Melilotus & 30 & 4,9 \\
\hline & Légumineuses, indéterminées & Fabaceae & 102 & 16,5 \\
\hline \multirow{7}{*}{ Divers } & Amaranthe & Amaranthus sp. & 3 & 0,5 \\
\hline & Chenopodiacées & Chenopodiaceae & 2 & 0,3 \\
\hline & Mauve & Malva cf. M. parviflora & 1 & 0,2 \\
\hline & Palmier nain & Nannorrhops ritchieana & 5 & 0,8 \\
\hline & Jujubier & Ziziphus sp. & 1 & 0,2 \\
\hline & Indéterminés, fragments & - & 79 & 12,8 \\
\hline & & Total & 617 & 100,0 \\
\hline
\end{tabular}

céréales qui représentent près de $40 \%$ des restes identifiés. L'orge (Hordeum vulgare) est présente, mais le médiocre état de conservation des caryopses ne permet pas de déterminer s'il s'agit de l'orge vêtue ou de l'orge nue, cette dernière variété étant généralement plus courante sur les sites protohistoriques.

Deux espèces de blé ont été identifiées: le blé vêtu amidonnier (T. dicoccum) et le blé nu (Triticum durum/aestivum). Aucun autre élément de l'épi céréalier (segments de rachis glume, glumelle) n'est attesté dans les niveaux anciens.

Ces effectifs sont trop faibles pour permettre une interprétation des proportions entre les différents taxons. Néanmoins, la présence de l'amidonnier peut témoigner d'un certain «archaïsme». En effet, les blés vêtus constituent avec l'orge les premières céréales domestiquées dans le Croissant fertile et elles dominent généralement les assemblages archéobotaniques des sites néolithiques sur l'ensemble du
Moyen-Orient. Leur importance dans les économies agricoles diminue par la suite en faveur des blés nus de type hexaploïde qui deviennent prédominants dès le IV millénaire av. notre ère, dans la vallée de la Kech comme dans l'Est iranien et en Asie centrale.

Les céréales étaient très vraisemblablement cultivées dans un système de décrue où les semailles s'effectuaient après la récession de l'inondation de la Kech, dans les limons humides et fertiles apportés par la rivière. Ce type de cultures est encore pratiqué dans certaines vallées alluviales du Makran pakistanais, notamment dans le Dasht, où la topographie rend difficile l'utilisation des galeries souterraines drainantes (des kareez) qui approvisionnent actuellement les cultures en palmeraie des vallées plus étroites.

Deux autres espèces cultivées apparaissent à la période II (ca. 4000-3500 av. notre ère) de Shahi-Tump: la lentille (Lens 
culinaris) et le lin (Linum usitatissimum) ${ }^{22}$. Leur absence des niveaux les plus anciens ne traduit pas nécessairement une absence réelle de la phase néolithique acéramique. En effet, on remarque sur beaucoup de sites une préservation différentielle où certaines espèces sont rarement préservées, soit parce qu'elles rentrent peu en contact direct avec le feu lors de leur préparation, soit en raison de leur nature, comme le lin par exemple, dont le contenu oléagineux se détruit lors de l'exposition à la chaleur.

\section{EXPLOITATION DES RESSOURCES VÉGÉTALES SAUVAGES}

Les apports alimentaires des plantes cultivées étaient complétés par la cueillette de fruits sauvages. L'endocarpe ligneux (le noyau) d'un fruit de jujubier (Ziziphus sp.) est attesté dans un des échantillons du chantier I. Deux espèces de ce genre sont indigènes dans les confins indo-iraniens: Z. spina-christi (litt. «épine du Christ»), un arbre caractérisant les formations ouvertes de type pseudo-savane et $Z$. nummularia, un arbuste épineux. Les deux produisent des fruits (des drupes) comestibles, dont les restes sont retrouvés sur de nombreux sites archéologiques au Moyen-Orient ${ }^{23}$.

Les fruits carbonisés du palmier nain (Nannorrhops ritchieana) ont été récupérés de plusieurs contextes dans les niveaux anciens des chantiers I et III. Ce petit arbuste, aux feuilles palmées et au stipe réduit, fournit, outre des fruits, des feuilles fréquemment utilisées pour la fabrication de vanneries, nattes, etc. De nombreuses empreintes de nattes, attestées dans les niveaux plus récents (période II) à Shahi-Tump, pourraient témoigner d'une telle utilisation.

Le palmier nain et le jujubier sont des espèces résistantes à la sécheresse, poussant le plus souvent sur des sols pierreux, nombreux dans la vallée de la Kech, à proximité de la rivière et des sites.

Finalement, les échantillons de Shahi-Tump contiennent une douzaine d'autres taxons sauvages, appartenant pour la plupart aux familles des graminées (Poaceae) et des légumineuses (Fabaceae). Leur incorporation dans les assemblages carbonisés s'explique le mieux par le nettoyage des récoltes; ces taxons correspondraient alors à des plantes adventices. Certains d'entre eux ont également pu être broutés par les animaux domestiques et arriver au contact du feu par l'utilisation du fumier comme combustible. Cependant, cette pratique,

22. HENRY, 2003.

23. COSTANTINI and AUDISIO, 2001. attestée sur plusieurs sites archéologiques du Moyen-Orient ${ }^{24}$, concerne essentiellement des régions pauvres en ligneux, ce qui n'était pas le cas de la vallée de la Kech.

Les données archéobotaniques des niveaux inférieurs de Shahi-Tump sont moins riches que celles issues de l'étude archéozoologique, essentiellement pour des raisons de conservation. Néanmoins, elles suggèrent, à l'instar de la faune, une économie végétale fondée principalement sur des plantes cultivées.

\section{CONCLUSIONS}

En l'absence - ou quasi-absence - de chasse, toutes les données nous indiquent pourtant une société sans crises alimentaires majeures. On peut noter une maîtrise efficace de la domestication animale et végétale, même si manquent encore des espèces que l'on connaîtra par la suite (comme Ovis aries, le mouton), qui ne semblent pas avoir été exploitées durant la période I du site. La vallée de la Kech, au niveau de Turbat, à l'exception des petits territoires cultivés à proximité immédiate du site, devait alors ressembler aux «jungles» qui bordent tous les puissants cours d'eau d'Asie centrale, depuis l'Iran oriental jusqu'aux piémonts de la lointaine Sogdiane. Les résultats de l'analyse anthracologique de Shahi-Tump et du site voisin de Miri Qalat montrent effectivement la présence, aux périodes protohistoriques, d'importantes formations ripicoles le long de la Kech ${ }^{25}$.

Toute période de disette aurait, sans l'ombre d'un doute, entraîné une augmentation de la chasse, de la pêche et de tous les moyens de piégeage du moyen et petit gibier; les ossements de ces proies se dépisteraient alors à coup sûr dans les restes de faunes.

Par ailleurs, l'exploitation des ressources végétales, déjà ouverte à plusieurs variétés cultivées, procurait également d'importantes ressources en protéines végétales ${ }^{26}$. Cet ensemble de témoignages fournit l'image d'une population numériquement peu importante, et probablement sédentaire. En effet, des structures construites ont été dégagées sur les deux chantiers de la période I; mais, surtout, la présence du grand bétail ne peut pas être envisagée hors de la vallée humide de la Kech: les reliefs arides du Makran n'autorisent pas le développement de troupeaux de bœufs - pas plus que de moutons comme nous l'avons déjà noté. Un pastoralisme «latéral» de grande

\footnotetext{
24. MiLLER, 1984.

25. TENGBERG and THIÉBAULT, 2003.

26. HENRY, 2003.
} 
ampleur, le long de la Kech, comparable par exemple à celui des éleveurs peuls, serait incompatible avec le développement des surfaces cultivées. Si cette population relève bien d'une culture acéramique, elle en est alors très probablement l'un des ultimes exemples régionaux: la liste très restreinte des espèces domestiques exploitées, tant animales que végétales, en porte témoignage. Le bœuf, ainsi que la chèvre, sont des animaux qui ont été domestiqués depuis plusieurs millénaires par ailleurs (en particulier à Mehrgarh), et rien ne témoigne en quoi que ce soit d'une domestication, tant locale qu'aussi tardive. En revanche, les habitants de la période I de Shahi-Tump, en gérant leur petit et leur grand bétail à faible distance du site et de leurs cultures, nous apportent un témoignage original: celui de ne fournir aucune évidence d'un quelconque «stress» majeur par rapport à leur environnement.

Il est à souhaiter que de nouvelles autorisations de fouilles permettent bientôt à la Mission archéologique française au Makran de reprendre ses opérations à Shahi-Tump pour étendre les surfaces étudiables de la plus ancienne période d'occupation de ce site.

Jean DESSE

CNRS-UMR 6130 CEPAM

Université de Nice-Sophia Antipolis

250, rue Albert Einstein

06560 Valbonne - FRANCE

desse@cepam.cnrs.fr
Nathalie DESSE-BERSET CNRS-UMR 6130 CEPAM

Université de Nice-Sophia Antipolis 250, rue Albert Einstein 06560 Valbonne-FRANCE berset@cepam.cnrs.fr

Auréade HENRY CNRS-UMR 6130 CEPAM Université de Nice-Sophia Antipolis 250, rue Albert Einstein 06560 Valbonne - FRANCE henry@cepam.cnrs.fr

Margareta TENGBERG CNRS-UMR $7041 \mathrm{ArScAn}$ Maison de l'Archéologie et de l'Ethnologie 21, allée de l'Université 92023 Nanterre Cedex - FRANCE margareta.tengberg@mae.u-paris10.fr

Roland BESENVAL CNRS-UMR 9993

Centre de recherche archéologique Indus-Balochistan, Asie centrale et orientale 19, avenue d'Iéna 75016 Paris - FRANCE rolandbesenval@hotmail.com

\section{BIBLIOGRAPHIE}

BENECKE N. and NEEF R.

2001 Faunal and Plant Remains from Sohr Damb/Nal (Pakistan). South Asian Anthropology 2001: 83-91.

BESENVAL R.

1997 Entre le Sud-Est iranien et la plaine de l'Indus: le Kech-Makran. Recherches archéologiques sur le peuplement ancien d'une marche des confins iraniens. Arts asiatiques 52: 5-36.

2005 Chronology of Protohistoric Kech-Makran. In: JARRIGE C. and LEFEVRE V. (eds), South Asian Archaeology 2001: Proceedings of the Sixteenth International Conference of the European Association of South Asian Archaeologists (Paris, July 2001). Vol. I: Prehistory: 1-9. Paris: Éditions Recherche sur les Civilisations.

BESENVAL R. and SANLAVILle P.

1990 Cartography of Ancient Settlements in Central Southern Pakistani Makran: New Data. Mesopotamia XXV: 79-146.

BÖKÖNYI S.

1993 Domestication Models: The Anatolian-Mesopotamian and the others in Southwest Asia. In: BUITENHUIS H. and CLASON A.C. (eds), Archaeozoology of the Near East: 4-8. Leiden: Universal Book Service.

COSTANTINI L.

1984 The Beginning of Agriculture in the Kachi Plain: The Evidence of Mehrgarh. In: ALLCHIN B. (ed.), South Asian Archaeology: 29-33. Cambridge: Cambridge University Press.

COSTANTINI L. and AUDISIO P.

2001 Plant and Insect Remains from the Bronze Age Site of Ra's alJinz (RJ-2), Sultanate of Oman. Paléorient 26,1 : 143-156.

COSTANTINI L. and COSTANTINI-BIASINI L.

1985 Agriculture in Baluchistan between the 7th and the 3rd Millennium BC. Newsletter of Baluchistan Studies 2: 16-30.

DESSE J.

1997 Archéozoologie aux marges occidentales du Bélouchistan. In: KoKABI M. and WAHL J. (eds), Proceedings of the 7th ICAZ Conference (Constance, 26 sept.-1 oct. 1994): 671-676. Paris: L'Homme et l'animal (Anthropozoologica 25-26). 
DESSE J. et DESSE-BERSET N.

2005a Ancient Exploitation of Marine Resources on the Makran Coast (Balochistan, Pakistan). Proceedings of the Sixteenth International Conference on South Asian Archaeology (Paris, July 2001): 85-93. Paris: Éditions Recherche sur les Civilisations.

2005b Les Ichthyophages du Makran (Bélouchistan, Pakistan). Paléorient 31,1 : 89-100 (volume thématique coordonné par J. Desse et N. Desse-Berset, Anciennes exploitations des milieux aquatiques de l'Asie du Sud-Ouest, approches environnementales).

DESSE-BERSET N. et DESSE J.

2007 Exploitation du milieu marin par les populations du littoral au Bélouchistan (Pakistan et Iran), et leurs contacts avec les oasis de l'intérieur, dès la fin du Ve millénaire BC. In: HÜSTERPlugmann H. (ed.), The Role of Fish in Ancient Time. ICAZ International Council for Archaeozoology, 13th Fish Remains Working Group Meeting (Bâle, 5-9 oct. 2005): 133-142. Rahden/Westf.: Verlag Marie Leidorf GmbH (www. VML. de).

HENRY A

2003 Le tell de Shahi-Tump, vallée de la Kech, Makran, Pakistan. Graines, empreintes sur brique crue. Étude de macrorestes issus des chantiers I et II (saisons 1999-2000), IV $V^{e}-I I I^{e}$ millénaires av. J.-C. Mémoire de DEA non publié. Paris: Université de Paris X.

JARRIGE J.-F., JARRIGE C. and QUIVRON G.

2005 "Mehrgarh Neolithic: The Updated Sequence." In: JARRIGE C. and LefeVre V. (eds), South Asian Archaeology 2001: Proceedings of the Sixteenth International Conference of the European Association of South Asian Archaeologists (Paris, July 2001). Vol. I: Prehistory: 129-141. Paris: Éditions Recherche sur les Civilisations.

JARRIGE C., JARRIGE J.-F., MEADOW R. and QUIVRON G.

1996 Mehrgarh. Field Reports 1974-1985. From Neolithic Times to the Indus Civilization. The Reports of Eleven Seasons of Excavations in Kachi District, Balochistan by the French Archaeological Mission to Pakistan. Karachi: Department of Culture and Tourism, Government of Sindh, Pakistan, in Collaboration with the French Ministry of Foreign Affairs.

\section{LAMBERG-KARLOVSKY C.C. and BEALE T.W.}

1986 Excavations at Tepe Yahya, Iran 1967-1975: The Early Periods. Cambridge, Mass.: Peabody Museum of Archaeology and Ethnology, Harvard University (American School of Prehistoric Research Bulletin 38).
LAMBERG-KARLOVSKY C.C. and POTTS D.T. with contributions by PITTMAN H. and KOHL P.L.

2001 Excavations at Tepe Yahya, Iran 1967-1975. The Third Millennium. Cambridge, Mass.: Peabody Museum of Archaeology and Ethnology, Harvard University (American School of Prehistoric Research Bulletin 45).

MADELLA M. and FULLER D.Q.

2006 Palaeoecology and the Harappan Civilization of South Asia: A Reconsideration. Quaternary Science Reviews 25: 1283-1301.

MEADOW R.H

1981 Early Animal Domestication: A First Report of the Faunal Remains from Mehrgarh, Pakistan, South Asia. In: HÄRTEL H. (ed.), Papers from the Fifth International Conference of the Association of South-Asian Archaeologists in Western Europe held in the Museum für Indische Kunst der Staatlichen Museen Preussischer Kulturbesitz, Berlin: 143-179. Berlin: D. Reimer Verlag.

1986a Animal Exploitation in Prehistoric Southeastern Iran: Faunal Remains from Tepe Yahia and Tepe Gaz Tavila-R37, 55003000 BC. Unpublished PhD Dissertation. Cambridge, Mass.: Department of Anthropology, Harvard University.

1986b Chapter 3. The Geographical and Palaeoenvironmental Setting of Tepe Yahya. In: LAMBERG-KARLOVSKY C.C. and BEALE T.W. (eds), Excavations at Tepe Yahya, Iran. 1967-1975. The Early Periods : 21-38. Cambridge, Mass.: Peabody Museum of Archaeology and Ethnology, Harvard University (American School of Prehistoric Research Bulletin 38).

1993 Animal Domestication in the Middle East: A Revised View from the Eastern Margin. Paper 27. In: POSSEHL G.L. (ed.), Harappan Civilization: 295-320. New Dehli: Oxford and IBH (2nd ed.).

MILLER N.F.

1984 The Use of Dung as Fuel: An Ethnographic Example and an Archaeological Application. Paléorient 10,2: 71-79.

STEIN A.

1931 An Archaeological Tour in Gedrosia. Calcutta (Memoirs of the Archaeological Survey of India 43).

1937 Archaeological Reconnaissances in North-Western India and South-Eastern Iran. London: Macmillan \& Co.

TENGBERG M. and THIÉBAULt S

2003 Vegetation History and Wood Exploitation in Pakistani Baluchistan from the Neolithic to the Harappan period: The Evidence from Charcoal Analysis. In: WEBER S.A., BELCHER W.R. (eds), Indus Ethnobiology. New Perspectives from the Field: 21-63. Lanham, Oxford: Lexington Books. 


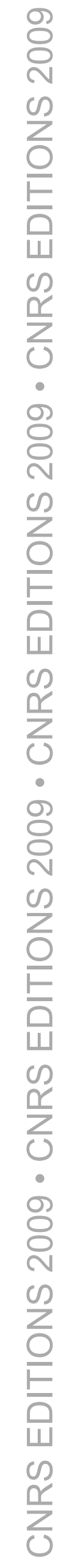

